\title{
A paper-based polystyrene/nylon Janus platform for the microextraction of UV filters in water samples as proof-of-concept
}

\author{
Juan L. Benedé ${ }^{1,2} \mathbb{D} \cdot$ Alberto Chisvert $^{2} \mathbb{E} \cdot$ Rafael Lucena $^{1} \mathbb{D} \cdot$ Soledad Cárdenas $^{1}(\mathbb{D}$
}

Received: 4 August 2021 / Accepted: 4 October 2021 / Published online: 25 October 2021

(c) The Author(s) 2021

\begin{abstract}
A new mix-mode cellulose-based sorptive phase is described that combines two different polymeric domains (i.e., nylon and polystyrene), thus providing simultaneous hydrophilic and hydrophobic features as a result. By analogy with Janus materials, the new paper-based sorptive phase has been named paper-based polystyrene/nylon Janus-platform (P-Ps/Ny-JP). The main advantages of the proposed P-Ps/Ny-JP are the sustainability, simplicity in synthesis, and low cost of this extraction device. The main parameters affecting the synthesis (i.e., coating procedure and polymers proportion) have been studied, and the resulting material has been characterized via scanning electron microscopy and infrared spectroscopy. As a proof-of-concept, the simultaneous extraction of fourteen UV filters of a wide range of polarity, with $\log P$ values ranging from -0.234 to 16.129, from water samples and their determination by liquid chromatography-tandem mass spectrometry (LC-MS/MS) has been performed. The proposed methodology enables the determination of these chemicals with limits of detection from 12 to $71 \mathrm{ng} \mathrm{L}^{-1}$, and the precision, expressed as a relative standard deviation, was below $15 \%$. The extraction device was applied to the analysis of real water samples likely to contain UV filters (i.e., two private swimming pool water and one seawater) and the recovery values were in the range $73-121 \%$.
\end{abstract}

Keywords Environmental analysis · Janus platform $\cdot$ Mixed-mode chemistry platform $\cdot$ Cellulose paper-based microextraction $\cdot$ Sustainable synthesis $\cdot$ UV filters $\cdot$ LC-MS/MS

\section{Introduction}

The synthesis of new sorptive phases is one of the leading research lines in the field of microextraction techniques. Green analytical chemistry principles [1] suggest using renewable resources and natural materials in their design $[2,3]$. Paper-based sorptive phases have demonstrated their potential, which relies on the sustainability and low price of

Soledad Cárdenas

scardenas@uco.es

1 Affordable and Sustainable Sample Preparation (AS2P) Research Group, Departamento de Química Analítica, Instituto Universitario de Investigación en Química Fina y Nanoquímica IUNAN, Universidad de Córdoba, Campus de Rabanales, Edificio Marie Curie (anexo), E-14071 Córdoba, Spain

2 GICAPC Research Group, Department of Analytical Chemistry, University of Valencia, 46100 Burjassot, Valencia, Spain the material and the versatility of the coatings. These phases were reported for the first time by Hurtubise and coworkers in the 1990s [4, 5], but their potential in microextraction has remained almost unexplored for two decades. The flat shape of the paper, formed by the irregular packing of the cellulose fibers, makes it attractive in the thin-film microextraction technique $[6,7]$. Although cellulose structure may lead to consider paper as an inert material, studies from Meng et al. [8] and Diaz-Liñan et al. [9] have demonstrated the capacity of unmodified paper to extract analytes. The paper surface can be covalently modified [10-13] or physically coated with a sorptive phase [14-19] for better performance. In the latter approach, dip-coating and drop-casting have emerged as useful synthesis techniques. They have allowed the preparation of papers coated with polymers [14-16], nanomaterials [19], or their combination (nanocomposites) [17, 18]. Other approaches, like the partial carbonization of paper [20], have been recently proposed to boost the extraction capacity of raw paper. 
A high selectivity is desirable when one compound or a family of structurally related compounds are targeted. However, an excessive selectivity may drive to chemical information losses when substances covering a wide range of chemical properties are intended to be determined in the same analysis. This situation is typical in non-targeted analysis (e.g., untargeted metabolomics) [21] or when a group of chemicals having a different structure but similar effect/ use (e.g., endocrine disrupters, UV filters) is targeted. In such cases, the simultaneous and effective isolation of the analytes becomes challenging. In a previous article [22], we have demonstrated that the cellulose substrate and a polyamide coating can synergically contribute to the extraction capacity allowing the joint isolation of analytes with logarithms of octanol/water partition coefficient $\left(\log \mathrm{P}_{\mathrm{ow}}\right)$ in the range from 0.88 to 2.83 . However, for a wider polarity interval, a single sorbent phase would not be versatile enough.

Janus materials present an intentionally designed anisotropy that can affect their shape or chemical structure [23]. These materials, which can comprise particle and flat shapes, have found application in many fields [24, 25], including biomedicine [26]. In particular, Janus papers consist of a flat cellulose substrate where two or more chemically different domains are found $[27,28]$. In the analytical chemistry realm, Janus papers are especially interesting in electrochemistry, where the simultaneous measurement of several analytes in a single and miniaturized platform can be conducted [29, 30]. However, the use of Janus papers in sample preparation has not been explored yet. To fill this gap, in the present article, a mix-mode paper-based sorptive phase is presented. It combines two different polymeric domains (i.e., nylon and polystyrene), providing simultaneous hydrophilic and hydrophobic features as a result. By analogy with Janus materials, the new paper-based sorptive phase has been named paper-based polystyrene/nylon Janus platform (P-Ps/Ny-JP). As a proof-of-concept, the trace determination of fourteen organic UV filters of a very wide polarity range ( $\log P_{\text {ow }}$ values from -0.234 to 16.129 ) in environmental water samples was considered for showing the potential and capability of this new extraction platform.

UV filters, which are added not only to cosmetics but also to other manufactured products to prevent or reduce the harmful effects of solar radiation [31], can reach the environment by direct and/or indirect sources and are being (bio)accumulated in aquatic ecosystems and living organisms [32]. Due to the harmful effects induced by these compounds on the aquatic flora and fauna [33], the development of analytical methods sensitive enough to determine them in this type of sample is one of the foci of attention of environmental research in recent decades [34-36].

The approach proposed in this article operates under the floating sampling technology mode, similarly to bar adsorptive microextraction $(\mathrm{BA} \mu \mathrm{E})$, introduced by Nogueira's research group in 2010 [37]. In essence, this technique is based on the flotation of the sorptive phase in the sample, thanks to its lower density. Thus, the sorptive phase floats above the vortex caused by the stirring of a magnetic bar. Unlike previous modalities of the technique, in which the extraction phase was adhered to a cylindrical polyethylene or polypropylene tube using a double-sided adhesive tape, in the present work, the P-Ps/Ny-JP itself is used as the unique extraction device.

\section{Experimental}

\section{Reagents}

The following fourteen UV filters were selected as analytes: benzophenone-4 (BZ4) 99.9\% from Roig Farma S.A. (Terrassa, Spain); benzophenone-3 (BZ3) 98\% and ethylhexyl salicylate (EHS) 99\% from Sigma-Aldrich (Barcelona, Spain, www.sigmaaldrich.com); isoamyl p-methoxycinnamate (IMC) 99.3\% from Haarmann and Reimer (Parets del Vallés, Spain, www.symrise.com); phenylbenzimidazole sulfonic acid (PBSA) 99\% and 4-methylbenzylidene camphor (MBC) 99.7\% from Guinama S.L. (Valencia, Spain, www.guinama.com); octocrylene (OC) $>98 \%$ from $F$. Hoffmann-La Roche Ltd. (Basel, Switzerland, www.roche. com); ethylhexyl dimethyl PABA (EHDP) 100\%, ethylhexyl methoxycinnamate (EHMC) 99.8\%, and butyl methoxydibenzoylmethane (BMDM) 98\% from Merck (Darmstadt, Germany, www.merckmillipore.com); diethylhexyl butamido triazone (DEBT) 99\% from $3 \mathrm{~V}$ Iberia S.A. (Barcelona, Spain); ethylhexyltriazone (EHT) 100\% and diethylamino hydroxybenzoyl hexyl benzoate (DHHB) $99.8 \%$ from BASF (Barcelona, Spain, www.basf.com/es); and drometrizole trisiloxane (DTS) 99.9\% from L'Oréal (Paris, France). Their chemical structures and relevant information are given in Table S1. A stock solution containing $1000 \mu \mathrm{g} \mathrm{mL}^{-1}$ of both hydrophilic UV filters (i.e., BZ4 and PBSA) was prepared in deionized water. Likewise, a stock solution of the twelve lipophilic UV filters was prepared in methanol at an individual concentration of $1000 \mu \mathrm{g} \mathrm{mL}^{-1}$. Both stock solutions were stored at $4{ }^{\circ} \mathrm{C}$ in the dark. Working solutions to obtain the calibration plots were prepared by appropriate dilution in deionized water (Millipore Corp., Madrid, Spain) from a 500-ng $\mathrm{mL}^{-1}$ intermediate aqueous solution of all target analytes.

LC-MS grade methanol and LC-MS grade water from Panreac (Barcelona, Spain, www.itwreagents.com/iberia) were employed as solvents of the chromatographic mobile phase. Gradient-grade methanol from VWR chemicals (Fontenay-sous-Bois, France, es.vwr.com/store) was used as the elution solvent. 
Common filter paper (grammage: $73 \mathrm{~g} \mathrm{~m}^{-2}$, thickness: $0.17 \mathrm{~mm}$ ) from Filtros Anoia S.A. (Barcelona, Spain, fanoia. com), formic acid $\geq 99 \%$ from VWR chemicals, nylon-6 pellets from Sigma-Aldrich, polystyrene obtained from commercial yoghurt containers, and chloroform from Panreac (Barcelona, Spain) were used for the synthesis of the P-Ps/ Ny-JP.

\section{Apparatus}

Liquid chromatographic analyses were performed using an Agilent 1260 Infinity HPLC system (Agilent, Palo Alto, CA, USA) equipped with a binary high-pressure pump for mobile phase delivery and an autosampler. Identification and quantification of the analytes were performed on an Agilent 6420 triple quadrupole MS with electrospray source using the Agilent MassHunter Software (version B.06.00) for data analyses.

Magnetic stirrers from J.P. Selecta, S.A. (Barcelona, Spain) and a Vibramax 110 shaker from Heidolph Instruments (Schwabach, Germany) were used for the stirring during extraction and elution procedures, respectively.

Scanning electron microscope (SEM) images were obtained by using a JEOL JSM 7800F microscope (JEOL, Tokyo, Japan) at the Central Service for Research Support (SCAI) of the University of Córdoba. ATR-IR spectra were acquired with a Bruker Tensor 37 FT-IR spectrometer (Bruker Optik, GmbH, Ettlingen, Germany) equipped with a three internal reflections diamond ATR cell (Platinum ATR accessory, Bruker). Data collection and processing were done using the OPUS software package (Bruker, Ettlingen, Germany). Contact angle measurements were performed in a Ramé-hart Model 200 Standard Goniometer with DropImage Standard v2.3 equipped with an automated dispensing system at the Instituto de Ciencia Molecular (ICMol) of the University of Valencia.

\section{Sample preparation}

Water samples were taken from two private swimming pools and Las Arenas Beach (Valencia, Spain), and they were analyzed as it will be described further on. All samples were collected in 2.5-L topaz glass bottles. Before analysis, unfiltered water samples were sonicated for $15 \mathrm{~min}$ in order to lixiviate the analytes from particles following the procedure previously developed to determine the total content of UV filters in the samples (i.e., the soluble fraction plus particulate fraction) [38]. Water samples were then conveniently diluted, if necessary, with deionized water to decrease the effect of ionic strength, as detailed later, in such a way the conductivity of the sample reached a value $\leq$ ca. $25 \mathrm{~S} \mathrm{~cm}^{-1}$.

\section{Synthesis of the paper-based polystyrene/nylon Janus platform}

The Janus platform, based on a polystyrene/nylon-coated cellulosic sorptive phase, was prepared in a two-stage process. First, a filter paper segment $(2 \mathrm{~cm} \times 1 \mathrm{~cm})$ was dipped halfway twice into $10 \mathrm{~mL}$ of a $6 \% \mathrm{w} / \mathrm{v}$ nylon- 6 solution prepared in formic acid. After each dip, the solvent was completely evaporated at room temperature (ca. $10 \mathrm{~min}$ ). Then, the other half was dipped twice into $10 \mathrm{~mL}$ of a $3 \% \mathrm{w} / \mathrm{v}$ polystyrene solution prepared in chloroform. As before, the solvent was evaporated at room temperature between each dip (ca. $5 \mathrm{~min}$ ). The P-Ps/Ny-JP can be used for at least 2 weeks without loss of efficiency, and no additional cleaning step was required, as demonstrated in preliminary studies.

\section{Microextraction procedure}

For the extraction step, a P-Ps/Ny-JP was introduced in a beaker containing $200 \mathrm{~mL}$ of the aqueous standard solution or water sample. Then, the solution was magnetically stirred at $1000 \mathrm{rpm}$ for $30 \mathrm{~min}$ at room temperature. The extraction platform remained floating in the vortex generated by this stirring. After the extraction, the P-Ps/Ny-JP enriched with analytes was taken with clean tweezers, briefly rinsed by soaking it in deionized water, and dried at room temperature (ca. $5 \mathrm{~min}$ ). Then, it was transferred to a 5-mL vial, in which $1 \mathrm{~mL}$ of methanol was added ensuring its total immersion to carry out the elution step. After 10 min of stirring in a shaker, the methanolic extract was placed into an injection vial for LC-MS/MS analysis. Considering the simplicity and low cost of the synthesis, the P-Ps/Ny-JP was discarded after each analysis.

\section{Liquid chromatography-tandem mass spectrometry analysis}

Chromatographic separation was accomplished by reversedphase separation on a Zorbax SB-C18 $(50 \times 2.1 \mathrm{~mm}$ i.d., $1.8 \mu \mathrm{m})$ column from Agilent. An in-line filter $(0.2 \mu \mathrm{m}$, $2.1 \mathrm{~mm}$ i.d.), also from Agilent, was selected to preserve the integrity of the analytical column. The injection volume was $5 \mu \mathrm{L}$. The pumps supplied the following gradient at room temperature, being $0.1 \%$ aqueous formic acid (solvent A) and methanol (solvent B) the mobile phase: 0-1 min, $80 \%$ solvent B, 1-4 min linear gradient to $100 \%$ solvent B and held for $4 \mathrm{~min}$. The flow rate was $0.3 \mathrm{~mL} \mathrm{~min}{ }^{-1}$. The run time was $20 \mathrm{~min}$.

Mass spectrometer settings were fixed to improve the multiple reaction monitoring (MRM) signals. The detector operated in positive electrospray ionization mode $\left(\mathrm{ESI}^{+}\right)$for lipophilic UV filters and in negative electrospray ionization mode $\left(\mathrm{ESI}^{-}\right)$for hydrophilic UV filters. The flow rate and 
the temperature of the drying gas $\left(\mathrm{N}_{2}\right)$ were $11 \mathrm{~L} \mathrm{~min}^{-1}$ and $300{ }^{\circ} \mathrm{C}$, respectively. The nebulizer pressure was $15 \mathrm{psi}$, and the capillary voltage was kept at $6000 \mathrm{~V}$ in both polarity modes. Table S2 summarizes the MRM parameters for the analytes. The most intense $\mathrm{m} / \mathrm{z}$ ratio of the product ion (base peak) was selected as quantifier transition, and the second most intense product ion was selected as qualifier transition to ensure the identity of the analyte.

Figure S1 shows a chromatogram of an aqueous standard solution of the analytes at $500 \mathrm{ng} \mathrm{L}^{-1}$ subjected to the proposed method.

\section{Results and discussion}

A plethora of different analytical methodologies have been used for the determination of UV filters in environmental waters involving solid-phase microextraction approaches. Among the multitude of extraction phases used for the extraction of these compounds, polydimethylsiloxane (PDMS) stands out both in solid-phase microextraction (SPME) and stir bar sorptive extraction (SBSE) approaches [35]. However, this phase is only suitable for UV filters of low to medium polarity. Efforts have been carried out to extract the most polar UV filters but in a lesser extent, using polyethyleneglycol-based phases [39] or a nylon-based magnetic material [40], among others. Therefore, the development of new devices that allow the determination of all types of UV filters, both hydrophobic and hydrophilic, is interesting and necessary.

\section{Evaluation of the paper-based polystyrene/nylon Janus platform}

In order to evaluate the extraction ability and selectivity of the P-Ps/Ny-JP for a wide range of compounds of different polarities, fourteen UV filters with $\log P$ values from -0.234 (PBSA) to 16.129 (EHT) were selected as model compounds (Table S1). It is important to note that even though all these compounds can be cataloged within the same application family (i.e., UV filters), they belong to different chemical groups, namely, benzophenone derivatives (BZ3, BZ4 and DHHB), p-aminobenzoic derivatives (EHDP), salicylates (EHS), methoxycinnamates (EHMC and IMC), camphor derivatives $(\mathrm{MBC})$, triazine derivatives (EHT and DEBT), benzotriazole derivatives (DTS), benzimidazole derivatives (PBSA), and others (BMDM and OC). Additionally, among them, BZ4 and PBSA have a hydrophilic character and possess a negative charge in their structure due to the deprotonation of the sulfonate groups, whereas the remaining twelve exhibit lipophilic character. Therefore, and in terms of interaction chemistry with a sorbent phase, they cannot be jointly extracted with a high efficiency, as it is reported in the literature [35].

Firstly, the extraction capabilities of different cellulosebased supports for the extraction of all the analytes under study were compared. For this purpose, raw paper, nylon-coated paper (P-Ny), polystyrene-coated paper (P-Ps), and both polystyrene and nylon-coated paper (P-Ps/Ny) were used. These polymers were selected due to their affordability, easy preparation in solution, and expected interaction with analytes. In the synthesis of P-Ny and P-Ps supports, the paper $(1 \mathrm{~cm} \times 0.5 \mathrm{~cm})$ was dipped in each polymer solution (i.e., nylon in formic acid, and polystyrene in chloroform) twice, alternatively changing the direction of immersion $180^{\circ}$. In the case of P-Ps/Ny support, each polymer covered half of the paper $(2 \mathrm{~cm} \times 1 \mathrm{~cm})$. In all cases, the concentrations of the polymers were $3 \%(\mathrm{w} / \mathrm{v})$.

The extraction efficiency (EE, \%), calculated as the ratio of the amount of analyte extracted with respect to its initial amount in the donor phase, was used as response function. These experiments were accomplished by extracting $2 \mathrm{~mL}$ of aqueous standards at $50 \mathrm{ng} \mathrm{mL}^{-1}$ of the target analytes under non-optimized conditions. The stirring during extraction was maintained for $10 \mathrm{~min}$ at $1000 \mathrm{rpm}$. Elution of the analytes was performed with $1 \mathrm{~mL}$ of methanol, which was proven to be the best elution solvent in preliminary experiments, for $10 \mathrm{~min}$. The employed volume was high enough to cover the entire support in a $5-\mathrm{mL}$ vial.

As shown in the results depicted in Fig. 1, different trends were observed as a function of the polarity of the analytes. Thus, the two hydrophilic UV filters (i.e., PBSA and BZ4) were only extracted on P-Ny and P-Ps/Ny, probably due to the high affinity that sulfonated compounds have for polyamides such as nylon, as has been demonstrated in a previous work [40].

On the other hand, lipophilic compounds were, in general terms, more favorably extracted by using P-Ps support because of its hydrophobic nature, being this effect more pronounced when the polarity of the UV filters decreases. However, as mentioned above, the nylon was needed for the extraction of hydrophilic compounds, and therefore, the P-Ps support was discarded.

It should be mentioned that that the extraction through the P-Ps/Ny material allowed the extraction of all analytes, both hydrophilic and lipophilic, in a similar or even better way with respect to P-Ny. These results confirm the versatility and the complementary extraction of the P-Ps/Ny-JP for the simultaneous extraction of compounds of different polarity and chemical structures.

\section{Synthesis and characterization of the paper-based polystyrene/nylon Janus platform}

Once the extraction efficiency and usefulness of the P-Ps/ Ny-JP have been demonstrated, different factors that can 
Fig. 1 Comparison of extraction efficiencies of the analytes by using different extraction platforms (P-Ny, nylon-coated paper; P-Ps, polystyrene-coated paper; P-Ps/Ny, both polystyrene/nylon-coated paper) $(n=3)$

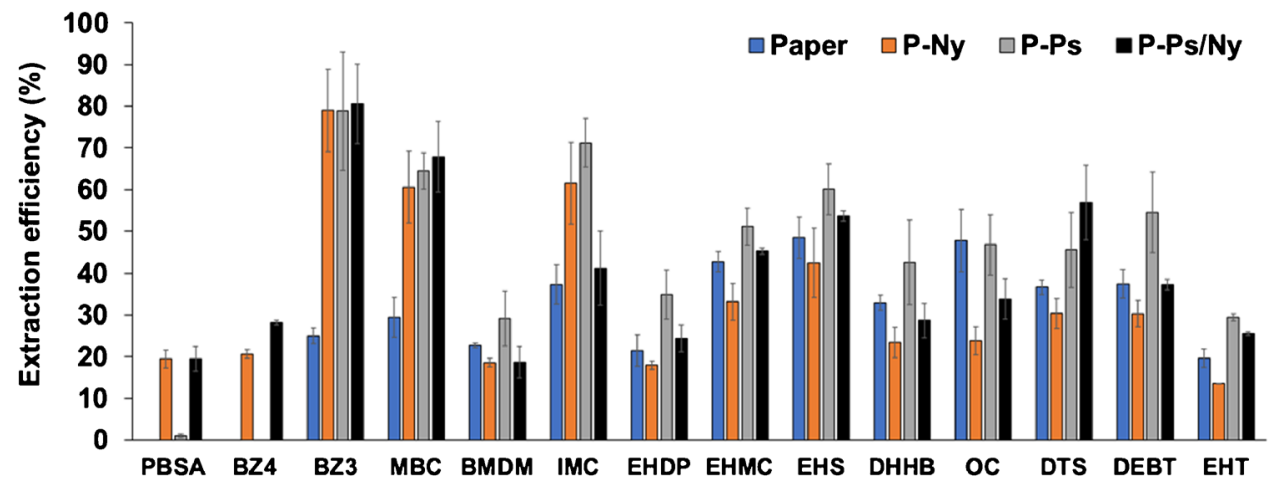

affect the synthesis of the support were evaluated. In this sense, the coating procedure and the polymers proportion were studied.

First, it was compared (1) dipping the paper $(2 \times 1 \mathrm{~cm})$ twice in a mixture of the two polymers $(3 \% \mathrm{w} / \mathrm{v})$ prepared in their respective solvents (i.e., nylon in formic acid and polystyrene in chloroform); and (2) coating the paper in halves (i.e., each polymer in one half of the paper), being both polymers at concentrations of $3 \%(\mathrm{w} / \mathrm{v})$. In the former case, the mixture was not homogeneous due to the immiscibility of the solvents. Therefore, it was necessary to shake the mixture before each dipping. The same extraction conditions described in the previous section were used. As can be seen in Fig. 2a, no significant differences were observed except for the two hydrophilic compounds (extracted by nylon), for which a better response was obtained when coating the paper in halves. This may be due to the fact that when the two polymers were previously mixed, the polystyrene also overlapped part of the nylon on the paper, thus preventing the interaction of these two compounds with the nylon. Thus, coating the paper in halves was adopted for the following studies.

Regarding the proportion of polymers, 1:1, 1:2 and 2:1 (polystyrene:nylon, Ps:Ny) were evaluated. The solutions of the polymers were prepared either at $3 \%(\mathrm{w} / \mathrm{v})$ or at $6 \%(\mathrm{w} / \mathrm{v})$ depending on the desired proportion of each polymer. Thus, the paper was always dipped in each polymer twice and the number of dips did not influence the extraction efficiency of the Janus platform. According to the results depicted in Fig. 2b, two trends were observed depending on the nature
Fig. 2 Comparison of extraction efficiencies for different a P-Ps/ Ny-JP synthesis procedures (1, dipping the platform twice in a mixture of the polymers; 2 , coating the platform with each polymer halfway) and $\mathbf{b}$ proportions of polystyrene and nylon, respectively, in the synthesis of the platforms
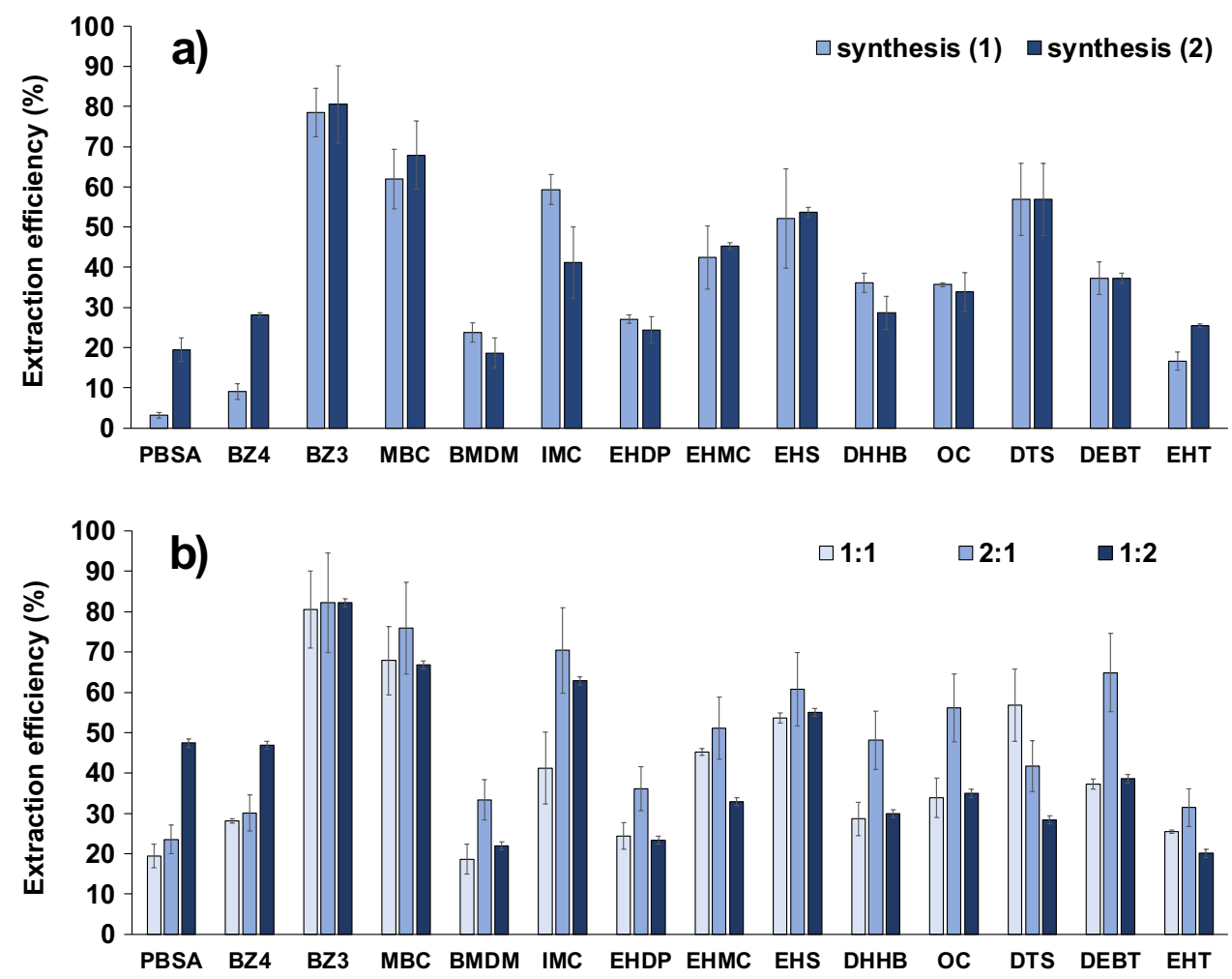
of the analytes. On the one hand, for those analytes extracted by nylon, better responses were obtained when the $1: 2$ ratio was used, and on the other hand, most lipophilic compounds were better extracted when the 2:1 ratio was used. To favor the extraction of the two hydrophilic compounds, the 1:2 Ps/Ny ratio was selected as optimal for the synthesis of the P-Ps/Ny-JP.

The Janus substrate was analyzed by SEM to study its morphology. As shown in Fig. 3a, the paper is composed of a non-uniform three-dimensional network of cellulosic fibers. Figure $3 b$ shows the partial coating of the paper surface by the nylon making it rougher, whereas the polystyrene is deposited on the paper more homogeneously, hiding the cellulose fibers of the paper (Fig. 3c). Finally, a detailed observation of the interface between nylon and polystyrene in the P-Ps/Ny-JP is shown in Fig. 3d, where both phases are clearly differentiated (i.e., the upper one is polystyrene and the lower one corresponds to nylon).

Likewise, the Janus material was also analyzed by ATRIR (Fig. S2). Nylon has characteristic and well-defined bands at $1537 \mathrm{~cm}^{-1}$ (NH deformation) and at $1633 \mathrm{~cm}^{-1}$ ( $\mathrm{C}=\mathrm{O}$ stretching). The main bands of paper are found at $2897 \mathrm{~cm}^{-1}$ (C-H symmetrical stretching), $3336 \mathrm{~cm}^{-1}$ (O-H stretching), and strong absorption in the range of $1033 \mathrm{~cm}^{-1}$ (C-O stretching), whereas polystyrene possesses defined bands at 694 and $746 \mathrm{~cm}^{-1}$ (C-H bending), and at 1450 and $1491 \mathrm{~cm}^{-1}$ (C-C stretching vibrations). In Fig. S2d and S2e, the characteristics peaks of paper and nylon and paper and polystyrene, respectively, are observed. The IR spectrum of the interface between nylon and polystyrene in the platform is presented in Fig. S2f, in which all the characteristic peaks and bands are observed, and thus indicating the success in the synthesis.

Finally, the contact angle of water on both parts of the support (i.e., nylon and polystyrene) was measured to show their different hydrophobicity. In the case of polystyrene side, an angle of $97.5^{\circ}$ was obtained (Fig. S3a), demonstrating its lipophilic character, whereas in the nylon side, the water drop was not maintained on the surface due to its hydrophilic character (Fig. S3b). Figure S3c shows a photograph of P-Ps/Ny-JP with a drop of water on each polymer.

\section{Optimization of the microextraction procedure using the paper-based polystyrene/nylon Janus platform}

Several critical variables (namely, sample volume, extraction time, elution time, $\mathrm{pH}$, and ionic strength) were considered in the optimization of the microextraction procedure. A univariate methodology was followed to understand the separate influence of each variable. In the initial conditions (i.e., non-optimized ones), aqueous standard solutions containing the fourteen UV filters at $50 \mathrm{ng} \mathrm{mL}^{-1}$ were extracted for $10 \mathrm{~min}$ and the analytes were eluted with $1 \mathrm{~mL}$ of methanol
Fig. 3 SEM images of a raw paper, b nylon-coated paper, c polystyrene-coated paper, and $\mathbf{d}$ interface of polystyrene/nylon in the P-Ps/Ny-JP, at magnification 60 a)

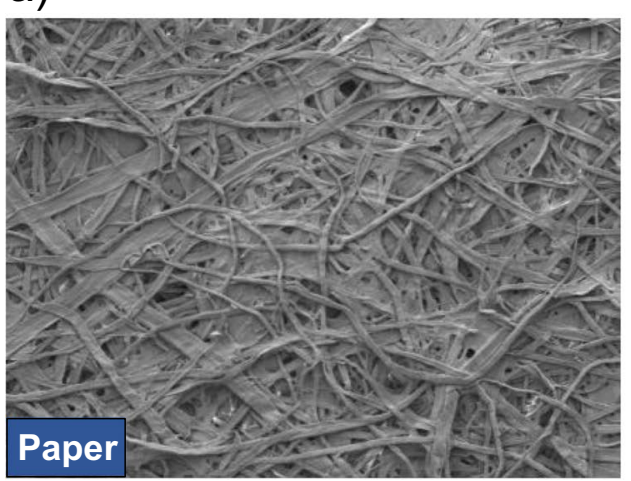

c)

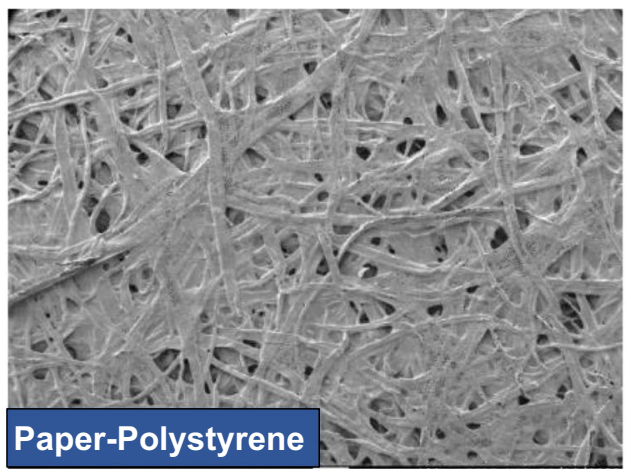

b)

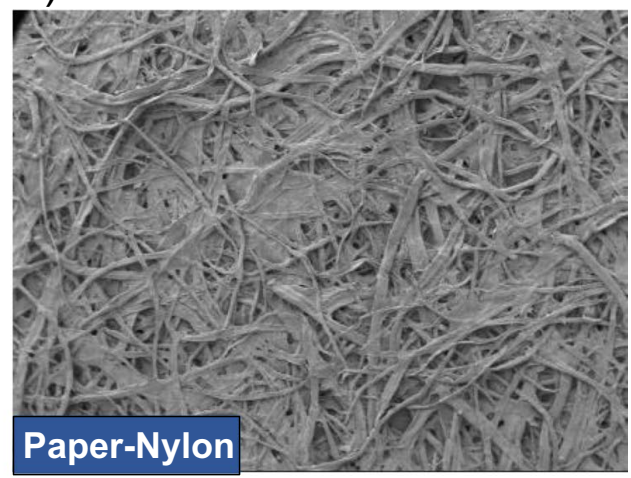

d)

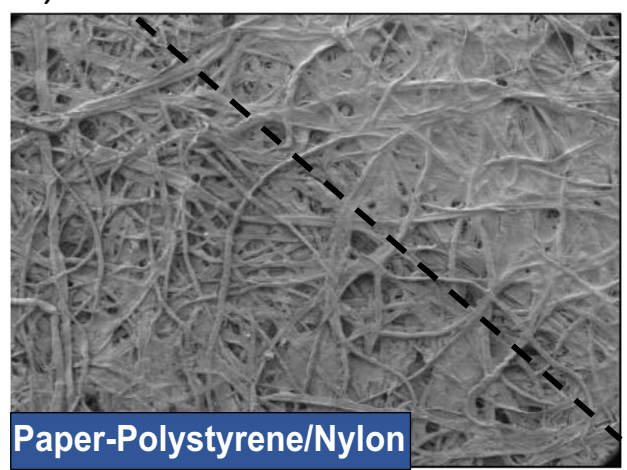


for $10 \mathrm{~min}$. The P-Ps/Ny-JP was synthesized as aforementioned. The enrichment factor (EF), determined as the ratio between the response of the aqueous standard solution after the extraction and the response obtained with the direct injection of this standard solution without extraction, was used as response function.

The results obtained for the optimization of sample volume, extraction time, elution time, and $\mathrm{pH}$ are included in the Supplementary Information (Fig. S4).

The effect of the ionic strength of the donor phase was assessed using sodium chloride as model electrolyte within the concentration range $0-10 \%(\mathrm{w} / \mathrm{v})$. These concentrations are equivalent to conductivity values from 0 to $160 \mathrm{~S} \mathrm{~cm}^{-1}$. The EFs decreased for all analytes, being more pronounced for values higher than $2 \%(\mathrm{w} / \mathrm{v})$, which can be ascribed to an increase of the water viscosity, thus hindering the transference of the analytes from the bulk sample to the extraction support. This fact can cause problems when seawater samples are analyzed, since their salt content can reach up $3 \%(\mathrm{w} / \mathrm{v})$. In order to prevent this problem, the dilution of the sample to reduce the influence of this variable on the recovery of the analytes was evaluated. As can be seen in Fig. S5, when dilution of an aqueous standard solution adjusted to $3 \%\left(\mathrm{NaCl}, \mathrm{w} / \mathrm{v}\right.$, conductivity ca. $\left.48 \mathrm{~S} \mathrm{~cm}^{-1}\right)$ was diluted with deionized water at 1:1 ratio (thus resulting in $1.5 \%(\mathrm{w} / \mathrm{v}$ ) of $\mathrm{NaCl}$ content), the EFs increased up to values similar to a standard without adjusting the ionic strength. Therefore, sample dilution of 1:1 was adopted for further analysis. These results showed that the conductivity should be measured, evaluated, and eventually reduced by sample dilution until it reaches a value $\leq \mathrm{ca} .25 \mathrm{~S} \mathrm{~cm}^{-1}$ when working with real samples, since the natural ionic strength of the sample may decrease the extraction of the analytes.

\section{Analytical figures of merit of the proposed method}

Once the variables that affect the extraction were selected, the main quality parameters of the proposed method were evaluated, namely, linearity, enrichment factors, limits of detection (LOD) and quantification (LOQ), and precision. The figures of merit are compiled in Table 1.

The linear range was evaluated using aqueous standard solutions at six concentration levels from 0.3 to $100 \mathrm{ng} \mathrm{mL}^{-1}$ for three UV filters (i.e., BZ4, EHS, and DTS) and from 0.1 to $100 \mathrm{ng} \mathrm{mL}^{-1}$ for the others UV filters, which were submitted to the microextraction procedure. Due to the low concentrations of these compounds expected in real water samples, the working calibration range was set from 100 or $300 \mathrm{ng} \mathrm{L}^{-1}$, depending on the analyte, to $1000 \mathrm{ng} \mathrm{L}^{-1}$, with $\mathrm{R}^{2} \geq 0.994$ in all cases. The estimated EFs were between 31 and 125, which corresponds to extraction efficiencies (EE) (calculated considering an $\mathrm{EF}_{\max }$ of 200) between 16 and $62 \%$. The LODs and LOQs were calculated as the concentration corresponding to a value of signal-to-noise of 3 and 10 , respectively. These values were found to be in the ranges 12-71 $\mathrm{ng} \mathrm{L}^{-1}$ and 41-238 $\mathrm{ng} \mathrm{L}^{-1}$, respectively, which agree with the EFs obtained. The precision of the method
Table 1 Main quality parameters of the proposed method

\begin{tabular}{|c|c|c|c|c|c|c|c|c|c|}
\hline \multirow[t]{3}{*}{ UV filter } & \multirow[t]{3}{*}{$\mathrm{EF}^{\mathrm{a}}$} & \multirow{3}{*}{$\begin{array}{l}\mathrm{EE}^{\mathrm{a}} \\
(\%)\end{array}$} & \multirow{3}{*}{$\begin{array}{l}\mathrm{LOD}^{\mathrm{b}} \\
\left(\mathrm{ng} \mathrm{L}^{-1}\right)\end{array}$} & \multirow{3}{*}{$\begin{array}{l}\mathrm{LOQ}^{\mathrm{b}} \\
\left(\mathrm{ng} \mathrm{L}^{-1}\right)\end{array}$} & \multicolumn{5}{|c|}{ Repeatability $(\% \text { RSD })^{\mathrm{c}}$} \\
\hline & & & & & \multicolumn{2}{|l|}{ Intra-day } & \multicolumn{3}{|c|}{ Inter-day } \\
\hline & & & & & $500 \mathrm{ng} \mathrm{L}^{-1}$ & $1000 \mathrm{ng} \mathrm{L}^{-1}$ & $500 \mathrm{n}$ & $\mathrm{g} \mathrm{L}^{-1}$ & $1000 \mathrm{ng} \mathrm{L}^{-1}$ \\
\hline PBSA & 82 & 41 & 28 & 93 & 12.7 & 7.8 & 14.7 & 12.3 & \\
\hline BZ4 & 36 & 18 & 56 & 185 & 13.1 & 9.7 & 8.1 & 11.1 & \\
\hline BZ3 & 64 & 32 & 20 & 67 & 10.2 & 10.1 & 10.7 & 12.6 & \\
\hline $\mathrm{MBC}$ & 54 & 27 & 29 & 98 & 8.1 & 6.1 & 7.3 & 11.1 & \\
\hline BMDM & 86 & 43 & 20 & 68 & 9.2 & 8.2 & 8.8 & 8.0 & \\
\hline IMC & 75 & 37 & 28 & 93 & 4.7 & 12.5 & 5.0 & 12.8 & \\
\hline EHDP & 61 & 30 & 22 & 74 & 5.3 & 12.2 & 7.5 & 9.6 & \\
\hline EHMC & 125 & 62 & 12 & 41 & 9.3 & 7.2 & 12.2 & 9.7 & \\
\hline EHS & 31 & 16 & 60 & 200 & 10.4 & 9.6 & 8.1 & 8.6 & \\
\hline DHHB & 110 & 55 & 22 & 75 & 13.0 & 8.8 & 11.1 & 13.9 & \\
\hline $\mathrm{OC}$ & 122 & 61 & 18 & 59 & 10.8 & 10.5 & 8.3 & 11.6 & \\
\hline DTS & 31 & 16 & 71 & 238 & 7.9 & 5.3 & 9.1 & 6.2 & \\
\hline DEBT & 95 & 48 & 26 & 86 & 5.8 & 2.7 & 7.6 & 8.9 & \\
\hline EHT & 45 & 22 & 27 & 91 & 9.0 & 10.4 & 11.9 & 11.5 & \\
\hline
\end{tabular}

${ }^{\text {a }} E F$ enrichment factor; $E E$ extraction efficiency

${ }^{\mathrm{b}} L O D$ limit of detection; $L O Q$ limit of quantification; calculated as 3 times and 10 times, respectively, the signal-to-noise ratio

${ }^{\mathrm{c}} R S D$ relative standard deviation 
Table 2 UV filters' contents in three water samples obtained by applying the proposed method $(n=3)$

\begin{tabular}{llll}
\hline UV filter & \multicolumn{2}{l}{ Found amount $\left(\mathrm{ng} \mathrm{L}^{-1}\right)$} \\
\cline { 2 - 4 } & Swimming pool 1 & Swimming pool 2 & Sea \\
\hline PBSA & $<$ LOD & $179 \pm 2$ & $<$ LOD \\
BZ4 & $540 \pm 40$ & $<$ LOD & $<$ LOD \\
BZ3 & $<$ LOD & $480 \pm 30$ & $704 \pm 70$ \\
MBC & $<$ LOD & $<$ LOD & $<$ LOD \\
BMDM & $281 \pm 2$ & $217 \pm 10$ & $815 \pm 80$ \\
IMC & $<$ LOD & $<$ LOD & $<$ LOD \\
EHDP & $<$ LOD & $<$ LOD & $<$ LOD \\
EHMC & $<$ LOD & $361 \pm 9$ & $595 \pm 60$ \\
EHS & $750 \pm 40$ & $<$ LOD & $600 \pm 20$ \\
DHHB & $<$ LOD & $210 \pm 10$ & $487 \pm 20$ \\
OC & $300 \pm 3$ & $335 \pm 7$ & $<$ LOD \\
DTS & $<$ LOD & $<$ LOQ & $<$ LOD \\
DEBT & $205 \pm 3$ & $137 \pm 10$ & $<$ LOD \\
EHT & $<$ LOD & $<$ LOD & $<$ LOD \\
\hline
\end{tabular}

was evaluated as relative standard deviation (RSD, \%) from the analysis of five different aqueous standard solutions containing the target analytes at 500 and $1000 \mathrm{ng}$ $\mathrm{L}^{-1}$ in the same day (intra-day repeatability) and in five different working sessions (inter-day repeatability). These values were between 4.7 and $14.7 \%$, thus showing the good precision of the method. The reproducibility of five P-Ps/Ny-JP prepared in different sessions was also studied. In this case, RSDs $<9.6 \%$ were obtained, thus demonstrating that the macroscopic variability that may exist between the different supports does not affect the repeatability of the extraction, and therefore the practical applicability is assured.

\section{Application to the analysis of water samples}

As a proof-of-concept of the P-Ps/Ny-JP, it was applied to the analysis of real water samples likely to contain UV filters: two private swimming pool water and one seawater. Furthermore, it should be noted that the salinity of these three samples was different, since one of the pools was treated water (swimming pool 1), and the other was saline (swimming pool 2). As previously mentioned, the conductivity of the samples was measured to estimate their saline content. Thus, the conductivity values for the two swimming pool water samples were 1.4 and $4.3 \mathrm{~S} \mathrm{~cm}^{-1}$, respectively (which correspond to approximate values of 0.08 and $0.2 \% \mathrm{NaCl}(\mathrm{w} / \mathrm{v}))$, while for the sea water sample, the conductivity value was $45.7 \mathrm{~S} \mathrm{~cm}^{-1}$ (ca. $2.9 \%$ of salt content). For this reason, the first two samples were analyzed without prior treatment, while the seawater sample was diluted with deionized water at ratio 1:1 prior to analysis (conductivity ca. $23 \mathrm{~S} \mathrm{~cm}^{-1}$ ). The samples were analyzed in triplicate according to the developed method. As can be seen in Table 2, all samples presented quantities of the target UV filters, predictably due to the use of sunscreens by bathers in these samples. It should be noted that the concentrations of these compounds, especially in seawater samples, depend on various factors such as the moment of sampling or the water tide, among others [41].

Finally, the reliability of the proposed method and the presence of matrix effects were evaluated by recovery studies. For this purpose, the three above-mentioned water samples were spiked with the target analytes at 500 and $1000 \mathrm{ng} \mathrm{L}^{-1}$, and subjected to the extraction process. The results presented in Table 3 shows that the obtained relative recoveries ranged from 81 to $121 \%$ in swimming pool waters and from 73 to $101 \%$ in sea water,
Table 3 Relative recoveries values obtained by applying the proposed method to three water samples spiked at two concentration level concentration $(n=3)$

\begin{tabular}{|c|c|c|c|c|c|c|}
\hline \multirow[t]{2}{*}{ UV filter } & \multicolumn{2}{|c|}{ Swimming pool 1} & \multicolumn{2}{|c|}{ Swimming pool 2} & \multicolumn{2}{|l|}{ Sea } \\
\hline & $500 \mathrm{ng} \mathrm{L}^{-1}$ & $1000 \mathrm{ng} \mathrm{L}^{-1}$ & $500 \mathrm{ng} \mathrm{L}^{-1}$ & $1000 \mathrm{ng} \mathrm{L}^{-1}$ & $500 \mathrm{ng} \mathrm{L}^{-1}$ & $1000 \mathrm{ng} \mathrm{L}^{-1}$ \\
\hline PBSA & $114 \pm 10$ & $119 \pm 3$ & $120 \pm 7$ & $114 \pm 10$ & $81 \pm 2$ & $90 \pm 7$ \\
\hline BZ4 & $106 \pm 10$ & $98 \pm 2$ & $98 \pm 10$ & $81 \pm 2$ & $86 \pm 10$ & $81 \pm 2$ \\
\hline BZ3 & $118 \pm 10$ & $107 \pm 5$ & $96 \pm 8$ & $83 \pm 2$ & $96 \pm 10$ & $84 \pm 2$ \\
\hline MBC & $105 \pm 5$ & $111 \pm 7$ & $103 \pm 10$ & $112 \pm 10$ & $86 \pm 9$ & $77 \pm 10$ \\
\hline BMDM & $105 \pm 10$ & $88 \pm 4$ & $115 \pm 5$ & $103 \pm 7$ & $99 \pm 5$ & $94 \pm 7$ \\
\hline IMC & $108 \pm 10$ & $94 \pm 10$ & $114 \pm 10$ & $103 \pm 7$ & $92 \pm 9$ & $82 \pm 6$ \\
\hline EHDP & $97 \pm 9$ & $114 \pm 3$ & $97 \pm 10$ & $113 \pm 2$ & $77 \pm 8$ & $90 \pm 2$ \\
\hline EHMC & $94 \pm 6$ & $119 \pm 5$ & $104 \pm 10$ & $84 \pm 2$ & $87 \pm 10$ & $82 \pm 2$ \\
\hline EHS & $108 \pm 10$ & $100 \pm 10$ & $84 \pm 8$ & $86 \pm 3$ & $84 \pm 20$ & $86 \pm 2$ \\
\hline DHHB & $96 \pm 10$ & $119 \pm 2$ & $115 \pm 2$ & $115 \pm 5$ & $101 \pm 4$ & $80 \pm 4$ \\
\hline $\mathrm{OC}$ & $97 \pm 2$ & $100 \pm 7$ & $101 \pm 2$ & $107 \pm 10$ & $95 \pm 2$ & $92 \pm 3$ \\
\hline DTS & $109 \pm 2$ & $113 \pm 6$ & $83 \pm 5$ & $98 \pm 8$ & $79 \pm 5$ & $96 \pm 8$ \\
\hline DEBT & $100 \pm 4$ & $98 \pm 2$ & $105 \pm 5$ & $121 \pm 3$ & $92 \pm 2$ & $80 \pm 2$ \\
\hline EHT & $116 \pm 10$ & $97 \pm 9$ & $109 \pm 8$ & $114 \pm 7$ & $73 \pm 5$ & $76 \pm 5$ \\
\hline
\end{tabular}




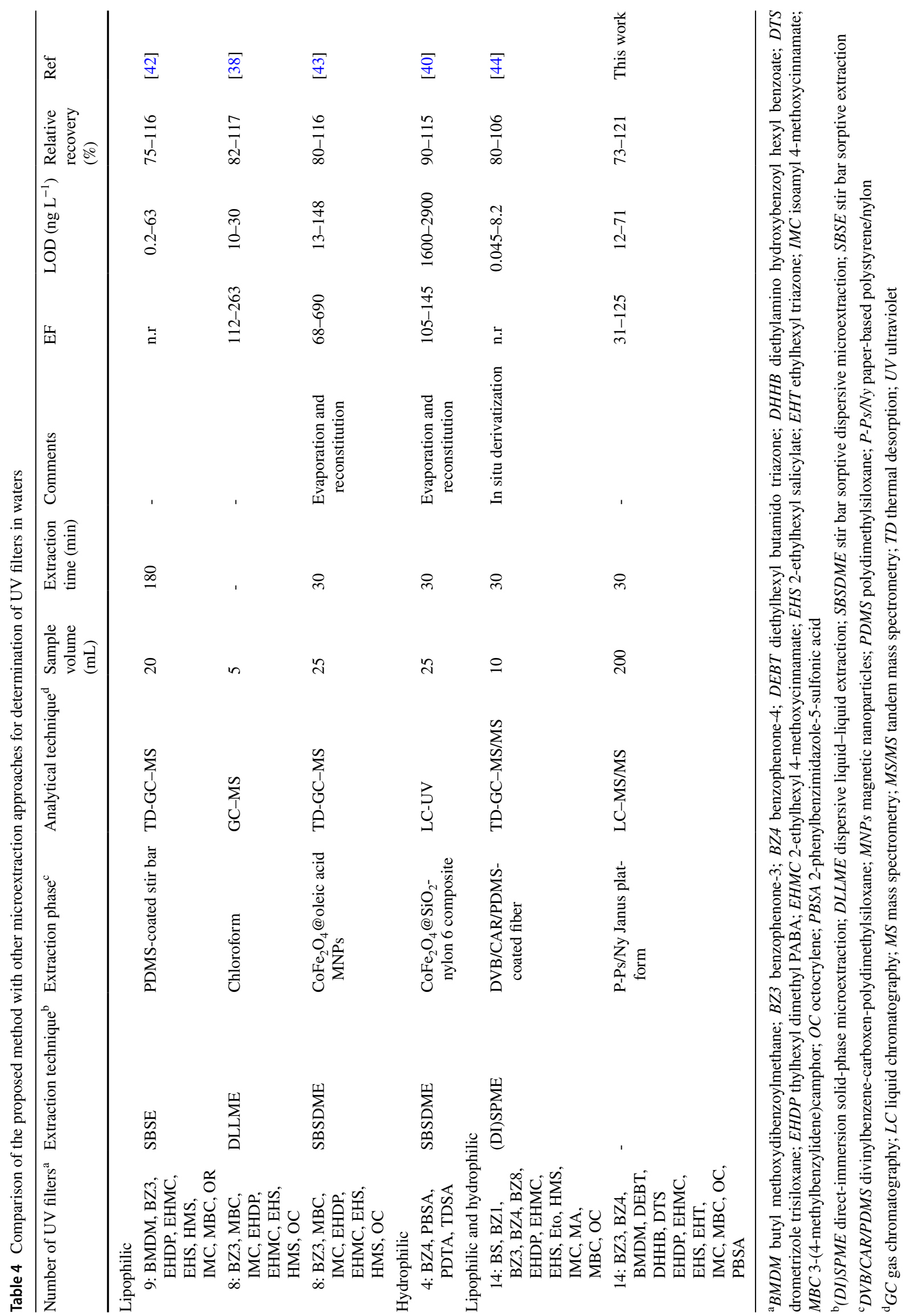


thus demonstrating the absence of matrix effects, beyond the effect of ionic strength in the seawater sample already considered and corrected by sample dilution.

\section{Comparison of the proposed method with other alternatives for determination of UV filters in waters}

The main analytical features of the proposed method were compared with other common microextraction approaches previously reported with the same purpose, covering the largest number of UV filters in common with this work (Table 4). Although the lowest LODs were not obtained by this method, they were in the low $\mathrm{n} \mathrm{L}^{-1}$ range, low enough to determine these compounds in this type of matrix, as demonstrated in the previous section. In any case, if necessary, the method can include a later step of evaporation of the elution solvent and subsequent reconstitution in a smaller volume, in order to further reduce the LOQs. Regarding accuracy, all studies presented relative recovery values higher than $70 \%$.

Nevertheless, the main advantages of the proposed P-Ps/ Ny-JP that deserve to be highlighted are the sustainability, simplicity in synthesis, and low cost of this extraction device. The starting materials used to prepare it (i.e., common filter paper, nylon, and polystyrene, which can even come from commercial packaging as in this article) are purchased in bulk, which is very competitive compared with SPME fibers or SBSE units subjected to commercial patents. In addition, the combination of different polymers offered a considerable improvement in the versatility and selectivity with respect to most approaches, which are mainly focused on the determination of lipophilic UV filters, in a simple way, allowing the simultaneous determination of a large number of UV filters, both hydrophilic and lipophilic. Additionally, no conditioning, derivatization or centrifugation steps are required in the proposed methodology, and no organochloride solvents are used during extraction procedure but only in the preparation of the support.

The main drawback of the proposed support is the independent and manual synthesis of each one, but remediable by automating the dip coating process, as well as the possibility of physical variation of the supports during synthesis, although it was shown that it did not significantly affect the repeatability in the extraction.

\section{Conclusions}

In the present article, a new and easy-to-prepare mix-mode cellulose-based sorptive phase with both hydrophilic and hydrophobic domains is presented. This Janus material is prepared using two affordable polymers such as nylon and polystyrene. The preparation of the extraction device only requires dipping the paper into organic solutions containing both polymers, and exhibits significant advantages, such as low cost and simple manipulation. The analytical potential of this new material is evaluated by the extraction of fourteen UV filters with very different polarity from waters. Although the polarity index (expressed as $\log \mathrm{P}_{\mathrm{ow}}$ values) ranged from -0.234 to 16.129 , the absolute extraction efficiencies varied between 16 and $62 \%$. These values, which are good for a microextraction procedure, provide enrichment factors in the interval 31-125 increasing the sensitivity of the analytical method. The advantage of being able to determine these UV filters of different polarity with different chemical structures by this Janus platform opens up new applications to extract many other families of compounds in a sustainable and economical way.

Supplementary Information The online version contains supplementary material available at https://doi.org/10.1007/s00604-021-05047-x.

Acknowledgements Financial support from the "Fondo Europeo de Desarrollo Regional (FEDER)" and Junta de Andalucía (Consejería de Transformación Económica, Industria, Conocimiento y Universidades, Project 1262884) is gratefully acknowledged. J.L.B would like to thank the Generalitat Valenciana and the European Social Fund for his postdoctoral grant. Funding for open access charge: Universidad de Córdoba / CBUA. This article is based upon work from the Spanish Thematic Network on Sample Treatment (RED-2018-102522-T), and the Sample Preparation Study Group and Network supported by the Division of Analytical Chemistry of the European Chemical Society.

Author contribution Juan L. Benedé: investigation, data curation, writing—original draft. Alberto Chisvert: supervision, writing — review and editing. Rafael Lucena: conceptualization, funding acquisition, writing—review and editing. Soledad Cárdenas: supervision, funding acquisition, writing—review and editing.

Funding Open Access funding provided thanks to the CRUECSIC agreement with Springer Nature.

\section{Declarations}

Conflict of interest The authors declare no competing interests.

Open Access This article is licensed under a Creative Commons Attribution 4.0 International License, which permits use, sharing, adaptation, distribution and reproduction in any medium or format, as long as you give appropriate credit to the original author(s) and the source, provide a link to the Creative Commons licence, and indicate if changes were made. The images or other third party material in this article are included in the article's Creative Commons licence, unless indicated otherwise in a credit line to the material. If material is not included in the article's Creative Commons licence and your intended use is not permitted by statutory regulation or exceeds the permitted use, you will need to obtain permission directly from the copyright holder. To view a copy of this licence, visit http://creativecommons.org/licenses/by/4.0/. 


\section{References}

1. Gałuszka A, Migaszewski Z, Namieśnik J (2013) The 12 principles of green analytical chemistry and the SIGNIFICANCE mnemonic of green analytical practices. TrAC - Trends Anal Chem 50:78-84. https://doi.org/10.1016/j.trac.2013.04.010

2. Godage NH, Gionfriddo E (2020) Use of natural sorbents as alternative and green extractive materials: a critical review. Anal Chim Acta 1125:187-200. https://doi.org/10.1016/j.aca.2020.05.045

3. Mafra G, García-Valverde MT, Millán-Santiago J et al (2020) Returning to nature for the design of sorptive phases in solidphase microextraction. Separations 7:1-22. https://doi.org/10. 3390/separations 7010002

4. Chen J, Hurtubise RJ (1998) Solid-phase microextraction with Whatman 1PS paper and direct room-temperature solid-matrix luminescence analysis. Talanta 45:1081-1087. https://doi.org/10. 1016/S0039-9140(97)00214-2

5. Ackerman AH, Hurtubise RJ (2002) Methods for coating filter paper for solid-phase microextraction with luminescence detection and characterization of the coated filter paper by infrared spectrometry. Anal Chim Acta 474:77-89. https://doi.org/10.1016/ S0003-2670(02)01002-4

6. Bruheim I, Liu X, Pawliszyn J (2003) Thin-film microextraction. Anal Chem 75:1002-1010

7. Olcer YA, Tascon M, Eroglu AE, Boyac1 E (2019) Thin film microextraction: towards faster and more sensitive microextraction. TrAC - Trends Anal Chem 113:93-101. https://doi.org/10. 1016/j.trac.2019.01.022

8. Meng X, Liu Q, Ding Y (2017) Paper-based solid-phase microextraction for analysis of 8-hydroxy-2'-deoxyguanosine in urine sample by CE-LIF. Electrophoresis 38:494-500. https://doi.org/ 10.1002/elps.201600439

9. Díaz-Liñán MC, Lucena R, Cárdenas S, López-Lorente AI (2021) Unmodified cellulose filter paper, a sustainable and affordable sorbent for the isolation of biogenic amines from beer samples. J Chromatogr A 1651:462297. https://doi.org/10.1016/j.chroma. 2021.462297

10. Saraji M, Farajmand B (2013) Chemically modified cellulose paper as a thin film microextraction phase. J Chromatogr A 1314:24-30. https://doi.org/10.1016/j.chroma.2013.09.018

11. Hashemian Z, Khayamian T, Saraji M (2014) Anticodeine aptamer immobilized on a Whatman cellulose paper for thin-film microextraction of codeine from urine followed by electrospray ionization ion mobility spectrometry. Anal Bioanal Chem 407:1615-1623. https://doi.org/10.1007/s00216-014-8392-5

12. Hashemi Hedeshi M, Rezvani O, Bagheri H (2020) Silane-based modified papers and their extractive phase roles in a microfluidic platform. Anal Chim Acta 1128:31-41. https://doi.org/10.1016/j. aca.2020.05.069

13. Ríos-Gómez J, García-Valverde MT, López-Lorente ÁI et al (2020) Polymeric ionic liquid immobilized onto paper as sorptive phase in microextraction. Anal Chim Acta 1094:47-56. https:// doi.org/10.1016/j.aca.2019.10.021

14. Ríos-Gómez J, Lucena R, Cárdenas S (2017) Paper supported polystyrene membranes for thin film microextraction. Microchem J 133:90-95. https://doi.org/10.1016/j.microc.2017.03.026

15. Díaz-Liñán MC, López-Lorente AI, Cárdenas S, Lucena R (2019) Molecularly imprinted paper-based analytical device obtained by a polymerization-free synthesis. Sensors Actuators B Chem 287:138-146. https://doi.org/10.1016/j.snb.2019.02.048

16. Díaz-Liñán MC, García-Valverde MT, Lucena R et al (2021) Dualtemplate molecularly imprinted paper for the determination of drugs of abuse in saliva samples by direct infusion mass spectrometry. Microchem J 160:105686. https://doi.org/10.1016/j.microc. 2020.105686
17. Casado-Carmona FA, Lucena R, Cárdenas S (2021) Magnetic paper-based sorptive phase for enhanced mass transference in stir membrane environmental samplers. Talanta 228:122217. https:// doi.org/10.1016/j.talanta.2021.122217

18. Díaz-Liñán MC, García-Valverde MT, López-Lorente AI et al (2020) Silver nanoflower-coated paper as dual substrate for surface-enhanced Raman spectroscopy and ambient pressure mass spectrometry analysis. Anal Bioanal Chem 412:3547-3557. https://doi.org/10.1007/s00216-020-02603-x

19. Ríos-Gómez J, Fresco-Cala B, García-Valverde MT et al (2018) Carbon nanohorn suprastructures on a paper support as a sorptive phase. Molecules 23:1252. https://doi.org/10.3390/molecules2 3061252

20. Bakhshizadeh Aghdam M, Farajzadeh MA, Afshar Mogaddam MR (2021) Partially carbonized cellulose filter paper as a green adsorbent for the extraction of pesticides from fruit juices. J Chromatogr A 1648:462220. https://doi.org/10.1016/j.chroma. 2021.462220

21. Sitnikov DG, Monnin CS, Vuckovic D (2016) Systematic assessment of seven solvent and solid-phase extraction methods for metabolomics analysis of human plasma by LC-MS. Sci Rep 6:1-11. https://doi.org/10.1038/srep38885

22. Benedé JL, Chisvert A, Lucena R, Cárdenas S (2021) Synergistic combination of polyamide-coated paper-based sorptive phase for the extraction of antibiotics in saliva. Anal Chim Acta 1164:338512. https://doi.org/10.1016/j.aca.2021.338512

23. Duan Y, Zhao X, Sun M, Hao H (2021) Research advances in the synthesis, application, assembly, and calculation of janus materials. Ind Eng Chem Res 60:1071-1095. https://doi.org/ 10.1021/acs.iecr.0c04304

24. Fan X, Yang J, Loh XJ, Li Z (2019) Polymeric Janus nanoparticles: recent advances in synthetic strategies, materials properties, and applications. Macromol Rapid Commun 40:1-19. https://doi.org/10.1002/marc.201800203

25. Koşak Söz Ç, Trosien S, Biesalski M (2018) Superhydrophobic hybrid paper sheets with Janus-type wettability. ACS Appl Mater Interfaces 10:37478-37488. https://doi.org/10.1021/ acsami.8b12116

26. Su H, Hurd Price CA, Jing L et al (2019) Janus particles: design, preparation, and biomedical applications. Mater Today Bio 4:100033. https://doi.org/10.1016/j.mtbio.2019.100033

27. Nau M, Herzog N, Schmidt J et al (2019) Janus-type hybrid paper membranes. Adv Mater Interfaces 6:1-8. https://doi.org/ 10.1002/admi.201900892

28. Go K, Bae K, Park K et al (2021) Janus black cellulose paper for fast volume reduction of liquid pollutant using solar steam generation. J Ind Eng Chem 94:166-172. https://doi.org/10. 1016/j.jiec.2020.10.034

29. Mettakoonpitak J, Volckens J, Henry CS (2020) Janus electrochemical paper-based analytical devices for metals detection in aerosol samples. Anal Chem 92:1439-1446. https://doi.org/10. 1021/acs.analchem.9b04632

30. Nantaphol S, Kava AA, Channon RB et al (2019) Janus electrochemistry: simultaneous electrochemical detection at multiple working conditions in a paper-based analytical device. Anal Chim Acta 1056:88-95. https://doi.org/10.1016/j.aca.2019.01. 026

31. Chisvert A, Salvador A (2018) Ultraviolet filters in cosmetics: regulatory aspects and analytical methods. In: Chisvert A, Salvador A (eds) Analysis of cosmetic products, 2nd edn. Elsevier B.V, pp 85-106

32. Cadena-Aizaga MI, Montesdeoca-Esponda S, Torres-Padrón ME et al (2020) Organic UV filters in marine environments: an update of analytical methodologies, occurrence and distribution. Trends Environ Anal Chem 25:e00079. https://doi.org/10.1016/j.teac. 2019.e00079 
33. Huang Y, Law JCF, Lam TK, Leung KSY (2021) Risks of organic UV filters: a review of environmental and human health concern studies. Sci Total Environ 755:142486. https://doi.org/10.1016/j. scitotenv.2020.142486

34. Chisvert A, Benedé JL, Salvador A (2018) Current trends on the determination of organic UV filters in environmental water samples based on microextraction techniques - a review. Anal Chim Acta 1034:22-38. https://doi.org/10.1016/j.aca.2018.05.059

35. Chisvert A, Giokas D, Benedé JL, Salvador A (2018) Environmental monitoring of cosmetic ingredients. In: Chisvert A, Salvador A (eds) Analysis of cosmetic products, 2nd edn. Elsevier B.V, pp 435-547

36. Ramos S, Homem V, Alves A, Santos L (2015) Advances in analytical methods and occurrence of organic UV-filters in the environment - A review. Sci Total Environ 526:278-311. https://doi. org/10.1016/j.scitotenv.2015.04.055

37. Neng NR, Silva ARM, Nogueira JMF (2010) Adsorptive microextraction techniques-Novel analytical tools for trace levels of polar solutes in aqueous media. J Chromatogr A 1217:7303-7310. https://doi.org/10.1016/j.chroma.2010.09.048

38. Benedé JL, Chisvert A, Salvador A et al (2014) Determination of UV filters in both soluble and particulate fractions of seawaters by dispersive liquid-liquid microextraction followed by gas chromatography-mass spectrometry. Anal Chim Acta 812:50-58. https://doi.org/10.1016/j.aca.2013.12.033

39. Gilart N, Miralles N, Marcé RM et al (2013) Novel coatings for stir bar sorptive extraction to determine pharmaceuticals and personal care products in environmental waters by liquid chromatography and tandem mass spectrometry. Anal Chim Acta 774:51-60. https://doi.org/10.1016/j.aca.2013.03.010
40. Benedé JL, Chisvert A, Giokas DL, Salvador A (2016) Stir bar sorptive-dispersive microextraction mediated by magnetic nanoparticles-nylon 6 composite for the extraction of hydrophilic organic compounds in aqueous media. Anal Chim Acta 926:6371. https://doi.org/10.1016/j.aca.2016.04.042

41. Tovar-Sánchez A, Sánchez-Quiles D, Basterretxea G et al (2013) Sunscreen Products as Emerging Pollutants to Coastal Waters. PLoS ONE 8:e65451. https://doi.org/10.1371/journal.pone.0065451

42. Rodil R, Moeder M (2008) Development of a method for the determination of UV filters in water samples using stir bar sorptive extraction and thermal desorption-gas chromatography-mass spectrometry. J Chromatogr A 1179:81-88. https://doi.org/10. 1016/j.chroma.2007.11.090

43. Benedé JL, Chisvert A, Giokas DL, Salvador A (2014) Development of stir bar sorptive-dispersive microextraction mediated by magnetic nanoparticles and its analytical application to the determination of hydrophobic organic compounds in aqueous media. J Chromatogr A 1362:25-33. https://doi.org/10.1016/j.chroma. 2014.08.024

44. Vila M, Celeiro M, Lamas JP et al (2017) Simultaneous in-vial acetylation solid-phase microextraction followed by gas chromatography tandem mass spectrometry for the analysis of multiclass organic UV filters in water. J Hazard Mater 323:45-55. https://doi. org/10.1016/j.jhazmat.2016.06.056

Publisher's note Springer Nature remains neutral with regard to jurisdictional claims in published maps and institutional affiliations. 